\title{
Microbiology Specimen Derived Flag
}

National Cancer Institute

\section{Source}

National Cancer Institute. Microbiology Specimen Derived Flag. NCI Thesaurus. Code C87904.

An indication that a data element corresponding to a microbiology specimen has been derived from one or more data elements. 